\title{
High Voltage Subnanosecond Monocycle Active Former Based on Compression Energy Unit
}

\author{
M.R. Ulmaskulov*, S.A. Shunailov and K.A. Sharypov \\ Institute of Electrophysics, RAS, 106 Amundsen Str., Yekaterinburg, 620016, Russia
}

\begin{abstract}
Compact high voltage subnanosecond monocycle former (MF) was tested. The device was designed on base of energy compression device and operated in a travelling wave mode. MF consists of high impedance and forming lines, which discharged for the load in parallel. As a result, the monocycle with pulse duration $\sim 1$ ns and typical peak-to-peak amplitude $\sim 250 \mathrm{kV}$ was formed without additional peaking of the nanosecond pulse rise time. Some pulses reached the maximum amplitude $\sim 270 \mathrm{kV}$. The parallel discharge circuit, and a spiral shape of forming line allowed keeping small dimensions of $\mathrm{MF}$, and save enough electrical strength to operate with pulse repetition rate up to 100 pps.
\end{abstract}

PACS numbers: 84.40.Az, 84.70.+p, 84.90.+a

\section{Introduction}

High voltage active monocycle former (MF) in [2] allowed forming stable bipolar pulses with pulse duration $1 \mathrm{~ns}$ and peak-to-peak amplitude $250 \mathrm{kV}$ in the mode of preliminarily peaking of rise time of initial nanosecond pulse. For that an additional gas discharger was used, which deteriorated waveform stability, introduced additional energy losses and increased dimensions of device. Aim of this work was creating device without the mentioned disadvantages and to keep output pulse parameters. MF was realized on base of compression unit to solve the task.

\section{Principle of operation}

The principle of operation of MF is based on the commutation of charged forming line (FL) simultaneously from both sides by two dischargers. One of discharger commutates FL for a load and forms the first peak of pulse. The discharger from another side of FL short-circuits its central and shielding electrodes and forms second peak of pulse with opposite polarity. For shaping of pulses of subnanosecond time duration the device operated in a travelling wave mode and the process detailed in [2].

Device proposed in this work, like in [1], presents high voltage unit of energy compression and consists of forming and high impedance lines (FL and HL) with wave impedances $Z_{\mathrm{FL}}$ and $Z_{\mathrm{HL}}$ with divisible delay times $\left(t_{\mathrm{HL}}, t_{\mathrm{FL}}\right)$, which were connected in series as shown in Fig. 1a. Charging of FL with an increase in the peak

\footnotetext{
* corresponding author; e-mail: marat@iep.uran.ru
}

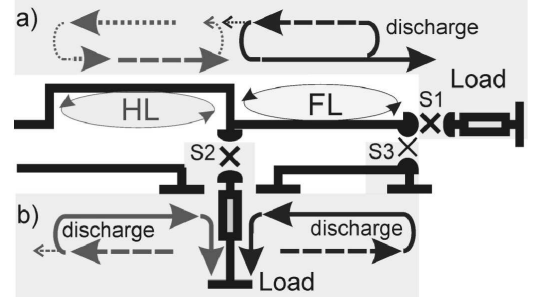

Fig. 1. Schemes of devices with (a) serial SD and (b) parallel PD discharge circuits.

power occurs in the travelling wave mode when HL and FL were connected to initial generator $\left(Z_{0}\right)$ in series, see Refs. $[1,3]$.

In this work the version of the scheme where the load $Z_{\text {load }}$ was connected to junction point of HL and FL by discharger S2 is proposed and S1 is absent correspondingly. In this case FL and HL discharge for the same load on the parallel electrical circuit (PD) (Fig. 1b) and form the part of pulse, whose polarity coincides with the polarity of initial nanosecond pulse. Scheme based on PD in contrast to serial (SD) connection of elements FL, HL and Load [1], made it possible to transmit into the load the energy, accumulated not only in FL, but also large part of the HL energy and to increase the energy effectiveness of device. Similar discharge mode of two parallel lines for a load was presented in our previous work [4].

Discharger S3 from another end of FL short-circuits its central and external electrodes, forms the opposite polarity part of the monocycle pulse. In this case FL and HL discharge for the load with different polarity voltage, which can lead to asymmetry shape of monocycle pulse. In order to ensure identical duration of both peaks of 


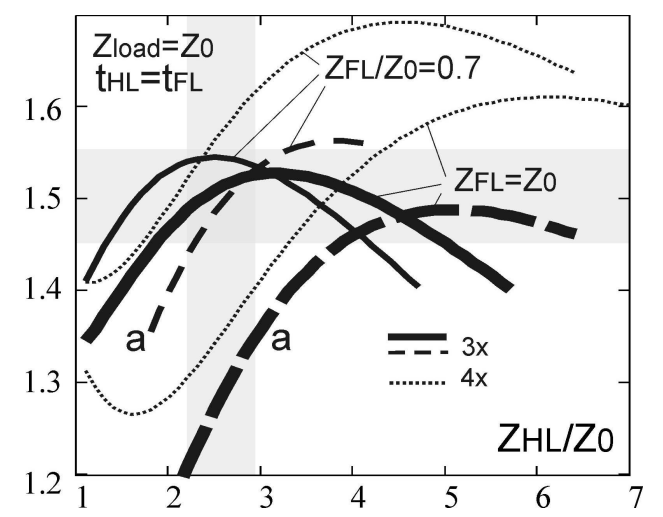

Fig. 2. Plots of voltages on a load for $\boldsymbol{a}$-triple compression of SD and 3 times and 4 times compressions of PD schemes.

monocycle pulse the dischargers S2 and S3 must operate simultaneously.

Results of the numerical comparison of the normalized voltage at the outputs of $\mathrm{SD}$ and $\mathrm{PD}$ schemes in the form of plots are presented in Fig. 2 for $t_{\mathrm{HL}}=t_{\mathrm{FL}}$. The plots of voltage multiplication factors for SD scheme on 3 times compressions (marked "a") and on 3 times and 4 times for PD scheme correspond to the condition $Z_{\text {load }}=Z_{\mathrm{FL}}=Z_{0}$. Plots confirm the possibility of increase of pulse peak voltage values by PD energy compression scheme in comparison with SD scheme under certain values $Z_{\mathrm{HL}} / Z_{0}$.

\section{Design}

The experimental model of converter with PD scheme with dischargers S2, S3 (Fig. 1b) with wave impedances $Z_{\text {load }}=Z_{\mathrm{FL}}=Z_{0}$ for $t_{\mathrm{HL}}=t_{\mathrm{FL}}$ was designed on basis of coaxial subnanosecond peaker [1] in case of adjustable nitrogen dischargers with the pressure up to 60 atmosphere. Dischargers operate stably when delay time to the moment of operation was less than 2.5-3 ns, which determined the multiplicity of pulse compression on the time not bigger than 4 for pulses with duration of $\sim 1 \mathrm{~ns}$.

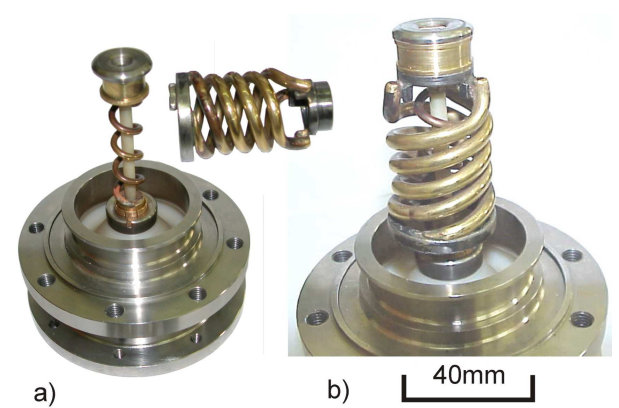

Fig. 3. Part of disassembled coaxial discharger without external electrode and output flange: (a) with installed HL and taken off FL, (b) assembling of FL and HL placed on insulator of an input flange of converter.
Forming line FL produced in form of three-winding spiral with estimated wave impedance $\sim 40-50 \Omega$ is shown in Fig. 3. The spiral-line was chosen for the purpose to decrease the overall size of device.

High impedance line HL was produced in form of usual single spiral-line with diameter less than that FL, but with identical length (Fig. 3). Scheme PD and fitted dimensions allowed to place HL inside of FL, thereby additionally decreasing the overall sizes of construction.

As load served oil line with impedance $45 \Omega$ with a capacity divider for control of pulse waveform.

\section{Experimental results}

As in the works $[1,2,4]$, as the driver of nanosecond pulses served the compact generator RADAN-303 [5], which in the given experiments formed across matched load $45 \Omega$ pulse with voltage amplitude $160 \mathrm{kV}$, pulse duration on the half-height $4.5 \mathrm{~ns}$ and rise time $\sim 1.5 \mathrm{~ns}$, with pulse repetition rate up to $100 \mathrm{~Hz}$.

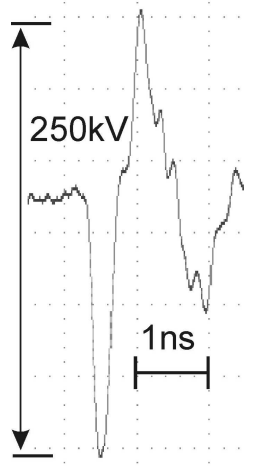

Fig. 4. Typical waveform of monocycle pulse.

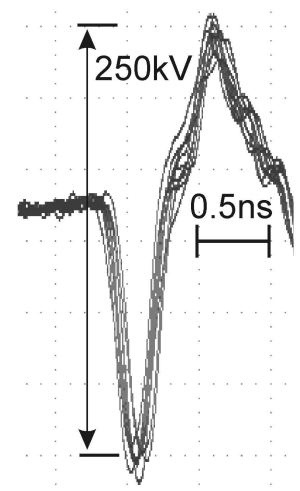

Fig. 5. Sampling of monocycle pulses waveforms generated in the single-shot mode.

Under operating only one discharger S2 the mono pulse was formed with FWHM $\sim 1.2$ ns and peak amplitude of voltage $\sim 210 \mathrm{kV}$. Shape of pulse was quasi-rectangular. It is obviously due to mismatching of spiral-line. When 
second discharger S3 switched on, the monocycle pulse with peak-to-peak amplitude $\sim 250 \mathrm{kV}$ and duration $\sim 1.1 \mathrm{~ns}$ was formed (Fig. 4). There is pulse behind the main pulse in the waveform with a lesser voltage amplitude, which is a consequence of mismatching of FL, HL and the load. Repetition of monocycle pulse waveform in a single-shot mode (Fig. 5) was enough high at experiment under stable of initial nanosecond pulse.

When device operated in the batch mode, at 500 pulses in packet and at repetition rate to $100 \mathrm{~Hz}$, signs of breakdown in the bipolar converter was not observed.

\section{Conclusions}

On the base of compression unit device with high-pressure gas discharger the compact subnanosecond high voltage former of monocycle pulses was designed. The device formed across load $\sim 50 \Omega$ monocycle pulses with duration $\sim 1 \mathrm{~ns}$ and typical peak-to-peak amplitude $\sim 250 \mathrm{kV}$.

The using both of the scheme of parallel discharge of FL and HL and spiral type waveguides allowed us to decrease the overall size of device in comparison with [2], with the retention of electric strength of device, sufficient for operation in the batch mode (at 500 pulses) with the pulse repetitive rate to $100 \mathrm{~Hz}$.

\section{References}

[1] V.G. Shpak, M.R. Oulmascoulov, S.A. Shunailov, M.I. Yalandin, in: Proc. 12th International IEEE Pulsed Power Conference, Monterey, CA, USA, 1999, p. 692 .

[2] V.G. Shpak, M.R. Oulmascoulov, S.A. Shunailov, M.I. Yalandin, in Proc. 12th International IEEE Pulsed Power Conference, Monterey, CA, USA, 1999, p. 1456.

[3] L.A. Morugin, G.V. Glebovich, Nanosecond Pulsed Technique, Sov. Radio, Moscow 1964, p. 623.

[4] V.G. Shpak, M.R. Ulmasculov, S.A. Shunailov, M.I. Yalandin, Izvestiy vuzov. Fizika, 2006, p. 305.

[5] V.G. Shpak, et al., Instrument Experimental Techniques (Russia), 36, 106 (1993). 